\title{
Operative Management and Outcomes of Patients with Sigmoid Volvulus at a Tertiary Public Hospital in Malawi
}

\author{
Ephraim Bitilinyu-Bangoh ${ }^{1}$, Fatsani Mwale ${ }^{1}$, Loveness Ulunji Chawinga ${ }^{1}$, Gift Mulima ${ }^{2}$ \\ ${ }^{1}$ College of Medicine, University of Malawi, Chichiri, Blantyre 3, Malawi. \\ ${ }^{2}$ Kamuzu Central Hospital, Lilongwe, Malawi
}

Correspondence to: Dr. Gift Mulima; Email: gmulima@gmail.com

Received: 20 Aug 2020; Revised: 16 Apr 2021; Accepted: 21 Apr 2021; Available online: 18 May 2021

\begin{abstract}
Background: Sigmoid Volvulus (SV) is a common cause of acute bowel obstruction in Malawi. We aimed to describe the surgical management of SV and its outcomes at Kamuzu Central Hospital, Lilongwe, Malawi. Methods: We retrospectively reviewed records from January 2019 to December 2019 of all SV patients, aged 18 years and above. Data extracted included age, sex, admission date, surgery date, bowel viability at time of surgery, procedure done, suspected anastomotic leakage, length of hospital stay and mortality. The data was analyzed using STATA 14.0. Results: There were more males $(\mathrm{n}=59,81.9 \%)$ than females. The median (IQR) age was 50.5 (38-60) years. A viable sigmoid colon was present in $61(84.7 \%)$ patients. The commonest procedures done were sigmoid resection and primary anastomosis (RPA) $(59.7 \%, \mathrm{n}=43)$ and Hartmann's procedure (HP) $(36.1 \%, n=26)$. The median length of hospital stay was 5 days in HP, 7 days in RPA
\end{abstract}

and longest in mesosigmoidopexy (10 days). Suspected anastomotic leakage occurred in $2(4.7 \%)$ patients. The overall mortality was $6.9 \%$ with all deaths occurring in RPA patients. Conclusion: Mortality is high in SV patients who undergo RPA. We recommend Hartmann's procedure in cases where the bowel has significant oedema or is gangrenous.

Keywords: sigmoid volvulus, resection and primary anastomosis, Hartmann's procedure,

mesosigmoidopexy.

Ann Afr Surg. 2021; 18(3): 176-179

DOI: http://dx.doi.org/10.4314/aas.v18i3.9

Conflicts of Interest: None

Funding: College of Medicine, University of Malawi, Student Research grants

(C) 2021 Author. This work is licensed under the Creative Commons Attribution 4.0 International License.

\section{Introduction}

Sigmoid volvulus (SV) is a life-threatening condition affecting more males than females in ratios ranging from 2:1 to 10:1 (1-3). It is more common in Eastern Europe, India and Africa, which account for $50 \%$ of all cases of intestinal obstruction (4). The mortality rate associated with SV is high, estimated in some series to be around $20 \%$ depending on treatment modality and case severity (5).

The precise etiology of SV remains speculative, and several etiological factors have been suggested including chronic constipation, a high fiber diet, bowel habits, high altitude, and enemas containing ginger, pepper and herbal extracts $(3,4,6)$. Classically, patients present with a triad of abdominal pain, constipation, and abdominal distention. An abdominal radiograph may reveal findings typical of volvulus; the coffee-bean sign, an inverted U-shape between the twisting and distending of the sigmoid colon and mesenteric axis in at least $60 \%$ of cases $(2,3)$.

Initial treatment of $\mathrm{SV}$ involves sigmoidoscopy with 
decompression and detorsion, with or without placement of a rectal tube. The success rate for sigmoidoscopic decompression can be between 60 and $81 \%(2,7,8)$. There are several surgical options that are considered to be acceptable treatment for SV including resection and primary anastomosis (RPA), Hartmann's procedure (HP), and mesosigmoidopexy depending on settings and resource availability (5).

In our study, we report our experience in the operative management and outcomes of SV patients at the Kamuzu Central Hospital (KCH), Lilongwe, Malawi. The hospital has a 1200 bed capacity and is the only tertiary and public referral hospital for the central region of the country (9).

\section{Methods}

We reviewed the admission and theater records of all patients with a diagnosis SV seen from January 1st 2019 to 31 st December 2019 aged 18 years and above. These were patients who had emergency surgery after failed decompression with rigid sigmoidoscopy, or decompression was not attempted at all, and those who had elective surgery after successful decompression and who were operated on within the same admission time period. Patients who were managed conservatively and those who had ileosigmoid knotting as a finding at surgery were excluded from the study. The primary outcome of the study was mortality.

The collected data were de-identified and the investigators had no access to the patient identifiers, that is, name and address. Variables of interest extracted from the records were age, sex, admission date, surgery date, sigmoid colon condition at time of surgery, that is, viable versus gangrenous (non-viable), and the ultimate procedure that was done during operation, that is, RPA or HP. The preoperative duration, defined as time (days) between admission to surgery, was calculated from the admission and surgery dates. Furthermore, we recorded suspected anastomotic leakage (SAL) as a complication in those who had RPA. Anastomotic leakage (AL) was suspected if observation of fecal material at the incision site or development of peritonitis was documented in the patient's file. The length of hospital stay (LOS) was also recorded.
Collected data were entered into Microsoft Excel 2016 MSO (16.0.4266.1001) and kept on a password protected computer. Analysis was done using STATA 14.0 (StataCorp, College Station, TX, USA). Descriptive statistics were used to determine rates of variables of interest. We set alpha at 0.05 . Fischer's exact test was used to describe the association between bowel viability and mortality among patients who had RPA.

\section{Ethical consideration}

The study was approved by the College of Medicine Research and Ethics Committee (COMREC), approval number U.06/19/2702, and the KCH Research Committee.

\section{Results}

A total of 85 patient records were collected and reviewed. Seventy-two cases met our study criteria and were analyzed. There were more males than females with a median [interquartile range (IQR)] age of 50.5 (38-60) years. Most patients had a viable sigmoid colon at time of operation. The commonest procedure done was a sigmoid resection and primary anastomosis. The median (IQR) preoperative duration was $0(0-8)$ days and median (IQR) LOS was 7 (5-8) days. The overall mortality was $6.9 \%$ (Table 1 ).

All patients who died $(\mathrm{n}=5,6.9 \%)$ were among those who had sigmoid resection and primary anastomosis ( $\mathrm{n}$ $=43,59.7 \%)$. Four $(90 \%)$ of these who died had viable bowel $(p=0.48)$. Two patients had SAL, one in each of the viable and non-viable bowel groups. No death was observed among those who had a HP or mesosigmoidopexy.

\section{Discussion}

The study has shown that the most common surgical procedures in the treatment of acute sigmoid volvulus at $\mathrm{KCH}$ are sigmoid colon RPA and HP. In 2010, Samuel et al. reported mesosigmoidopexy $(36 \%)$ as the most commonly performed procedure, followed by HP (33\%) and RPA (23\%) at KCH (5). The shift in the trend of type of procedures done may be explained by the development of a postgraduate surgery training program since $2009(10,11)$ which recruits trainees and produces 
surgeons almost yearly who may have different approaches to managing SV.

Table 1. Baseline patient characteristics and study findings.

\begin{tabular}{ll}
\hline VARIABLE & VALUES \\
\hline Age, median (range) & \\
Male $(n=59)$ & $52(42-63)$ \\
Female $(n=13)$ & $40(30-46)$ \\
Bowel condition, $n(\%)$ & \\
Viable sigmoid & $61(84.7)$ \\
Non-viable sigmoid & $11(15.3)$ \\
Procedure done, $n(\%)$ & \\
RPA & \\
Viable sigmoid & $4(59.7)$ \\
Non-viable sigmoid & $38(88.4)$ \\
Hartman's colostomy & $5(11.6)$ \\
Viable sigmoid & $26(36.1)$ \\
Non-viable sigmoid & $20(76.9)$ \\
Mesosigmoidopexy & $6(23.1)$ \\
Anastomotic leak, $n(\%)$ & $3(4.2)$ \\
Present & $n=43$ \\
Absent & $2(4.7)$ \\
LOS (days), median(range) & $41(95.3)$ \\
RPA & $7(1-21)$ \\
HP & $7(1-14)$ \\
Mesosigmoidopexy & $5(3-21)$ \\
Mean preoperative duration (days) $(\mathrm{SD})$ & $10(9-11)$ \\
Mortality $n$ (\%) & $12.4)$ \\
Discharge & \\
Death & \\
\hline & $67(93.1)$ \\
& $5(6.9)$ \\
\hline & \\
&
\end{tabular}

HP, Hartmann's procedure, LOS, length of stay, RPA, resection and primary anastomosis; SD, standard deviation.

There is no consensus with regard to the optimal surgical management in patients with an acute presentation. Some have advocated RPA as the treatment of choice for $\mathrm{SV}(4,6,12)$. As a general recommendation non-viable $\mathrm{SV}$ is best managed with HP. Viable SV is best managed with RPA, unless there are risk factors for AL, in which case the surgeon should consider mesosigmoidopexy with a non-absorbable suture (5).

Some studies in Africa report a low mortality, 3\%-6.4\%, in those patients undergoing emergency sigmoid RPA $(13,14)$. A higher overall mortality has been reported in a number of studies $(15,16)$. In our study we observed a mortality of $11.6 \%$ among RPA patients. This finding calls for a study to determine factors that may contribute to such a mortality rate among RPA patients in this setting.

The incidence of AL after colorectal surgery has been reported as $1 \%-30 \%$ in several studies (17). In a Mozambican study, Assan and Slivanov report a 5.5\% leak rate in $72 \mathrm{SV}$ patients who had an RPA (18). Similarly, low rates have been observed in the studies done in Malawi (7.2\%) and Kenya (4.5\%). In our study we report a suspected $4.7 \%(2 / 43)$ leak rate. The leak rate in our study may be higher than reported as leakage was a clinical diagnosis.

There was no mortality in patients who had HP or mesosigmoidopexy and the lowest LOS was observed with HP followed by an RPA. HP may be a safer procedure when mortality, anastomotic leakage, and LOS are considered. The longer LOS observed with mesosigmoidopexy suggests that these patients may have had clinical conditions that necessitated the procedure choice.

\section{Conclusion}

Mortality is high in SV patients who undergo bowel RPA. Performing this procedure should be done cautiously and after careful patient assessment. We recommend HP in cases where the bowel has significant edema or is gangrenous.

The limitations of our study are common with many retrospective studies; incomplete data in the file records and missing files of patients who were recorded to have been admitted with SV. In addition, we did not record symptom duration (time from symptom onset to presentation at $\mathrm{KCH}$ ) and risk factors for anastomotic failure to be included in the analysis.

\section{References}

1. Moseson DL, Lindell T, Brant B, et al. Sigmoid volvulus. Am Surg. 1976; 42(7): 492-497. 
2. Ifversen AKW, Kjaer DW. More patients should undergo surgery after sigmoid volvulus. World J Gastroenterol. 2014; 20(48): 18384-18389.

3. Chalya PL, Mabula JB. Sigmoid volvulus and ileo-sigmoid knotting: a five-year experience at a tertiary care hospital in Tanzania. World J Emerg Surg. 2015; 4-11.

4. Coban S, Yilmaz M, Terzi A, et al. Resection and primary anastomosis with or without modified blow-hole colostomy for sigmoid volvulus. World J Gastroenterol. 2008; 14(36): 5590-5594.

5. Samuel JC, Akinkuotu A, Msiska N, et al. Re-examining treatment strategies for sigmoid volvulus: An analysis of treatment and outcomes in Lilongwe, Malawi. Glob j Surg [Internet]. 2010;1(2):149-53. Available from: https://www.ncbi.nlm.nih.gov/pmc/articles/PMC334520/

6. Nasir M, Khan IALI. Resection and primary anastomosis in the management of acute sigmoid volvulus. Pakistan J Surg. 2014; 24(2): 95-99.

7. Lou Z, Yu E Da, Zhang W, et al. Appropriate treatment of acute sigmoid volvulus in the emergency setting. World J Gastroenterol. 2013; 19(30): 4979-4983.

8. Iida T, Nakagaki S, Satoh S, et al. Clinical outcomes of sigmoid colon volvulus: Identification of the factors associated with successful endoscopic detorsion. Intest Res. 2017; 15(2): 215-220.

9. Neuhann F, Barteit S. Lessons learnt from the MAGNET Malawian-German Hospital Partnership: The German perspective on contributions to patient care and capacity development. Global Health. 2017; 13(1): 1-14.

10. Kim SN. The Malawi Surgical Initiative at Kamuzu Central Hospital: A Program and Evaluation Plan. 2013:19.

11. Drønen SO.Training surgeons in Malawi: Traffic accidents are a huge burden on the health care system in Malawi. The University of Bergen (UiB) is a partner in a project to train more surgeons in Malawi. UiB Global; Developmentrelated Research. 2014:39.

12. Safioleas M, Chatziconstantinou C, Felekouras E, et al. Clinical considerations and therapeutic strategy for sigmoid volvulus in the elderly: A study of 33 cases. World J Gastroenterol. 2007;13(6):921-4.

13. Bhuiyan M, Machowski Z, Linyama B, et al. Management of sigmoid volvulus in Polokwane-Mankweng Hospital. S Afr J Surg. 2005; 43: 17-19.

14. Mulugeta GA, Awlachew S. Retrospective study on pattern and outcome of management of sigmoid volvulus at a district hospital in Ethiopia. BMC Surg. 2019; 19(1): $1-5$.

15. Nuhu A, Jah A. Acute sigmoid volvulus in a West African population. Ann Afr Med. 2010; 9:86-90.

16. Atamanalp SS. Sigmoid volvulus. EAJM 2010;42: 142147.

17. Zemenfes D, Tamirat E. Prevalence of and factors associated with anastomotic leakage among surgical patients at 2 teaching hospitals in Addis Ababa, Ethiopia. East Cent African J Surg. 2019; 24(2): 89-93.

18. Assan A, Slivanov I. Sigmoid volvulus: Management by resection and primary anastomosis. East Cent African J. 2001; 6(1): 19-20. 